\title{
Associação entre as características sociodemográficas e os tipos de exposição em portadores da Hepatite $\mathrm{C}$
}

Association between sociodemographic characteristics and exposure types in patients with Hepatitis C Asociación entre características sociodemográficas y tipos de exposición en pacientes con Hepatitis

Carla Fernanda Tiroli

ORCID: https://orcid.org/0000-0002-0974-9689

Universidade Estadual de Londrina, Brasil E-mail: carla_tiroli@yahoo.com.br

Rejane Kiyomi Furuya

ORCID: https://orcid.org/0000-0003-0885-5364 Instituto Federal do Paraná, Brasil

E-mail: re.furuya@gmail.com

Douglas Fernando Dias

ORCID: https://orcid.org/0000-0003-0266-4209 Universidade Estadual de Londrina, Brasil E-mail: df.dias@yahoo.com.br

Ligia Carla Faccin Galhardi ORCID: https://orcid.org/0000-0002-7522-9710 Universidade Estadual de Londrina, Brasil E-mail: lgalhardi@uel.br

Natacha Bolorino

ORCID: https://orcid.org/0000-0002-3039-2987 Universidade Estadual de Londrina, Brasil E-mail: natachabolorino@hotmail.com

Laís Cristina Gonçalves Ribeiro ORCID: https://orcid.org/0000-0002-4522-3297 Universidade Estadual de Londrina, Brasil E-mail: lcg.enf@hotmail.com Andressa Cristina Novaes ORCID: https://orcid.org/0000-0002-9298-5841 Universidade Estadual de Londrina, Brasil E-mail: andressa.novaes@ outlook.com

João Victor Rodrigues Cardoso ORCID: https://orcid.org/0000-0002-6505-7302 Universidade Estadual de Londrina, Brasil E-mail: joaoo.vrc@gmail.com

Marcos Augusto Moraes Arcoverde ORCID: https://orcid.org/0000-0001-5104-559X Universidade Estadual do Oeste do Paraná, Brasil E-mail: marcos.arcoverde2013@ gmail.com Flávia Meneguetti Pieri ORCID: https://orcid.org/0000-0003-1239-2550 Universidade Estadual de Londrina, Brasil E-mail: fpieri@uel.br

\begin{abstract}
Resumo
Objetivo foi de analisar a associação entre as características sociodemográficas e os tipos de exposição. Trata-se de um estudo transversal analítico e quantitativo, a partir de dados secundários das fichas de Hepatites Virais notificadas pelos municípios da 17 Regional de Saúde do Paraná de 2015 a 2018. Os dados foram analisados no Software Statistical Package for the Social Science. Na análise bivariada quanto na multivariável, as razões de prevalência e os intervalos de confiança de $95 \%$ foram calculados por meio da regressão de Poisson com ajuste robusto de variância e um nível de significância de 0,05. Na análise multivariável, notou-se associação estatisticamente significativa entre a variável sexo masculino e os seguintes tipos de exposição: drogas injetáveis, drogas inaláveis ou crack, medicamentos injetáveis e três ou mais parceiros sexuais. Em contrapartida, houve associação entre a faixa etária de 18 a 59 anos e
\end{abstract}


os seguintes tipos de exposição: drogas inaláveis ou crack, tatuagem e piercing, tratamento dentário e três ou mais parceiros. No grupo com baixa escolaridade, associação entre a escolaridade e os tipos de exposição, com menor prevalência, dos seguintes fatores: transfusional e tratamento dentário. Por fim, usar drogas inaláveis ou crack e ter três ou mais parceiros foram fatores de exposição para HCV associados ao sexo masculino e à faixa etária de 18 a 59 anos. O uso de drogas e o uso de medicamentos injetáveis foram fatores de exposição associados ao sexo masculino, enquanto tatuagem e piercing e tratamento dentário foram associados à faixa etária até 59 anos.

Palavras-chave: Hepatite C; Hepatite viral humana; Epidemiologia; Notificação de doenças; Saúde pública.

\begin{abstract}
Objectives was to analyze the association bewteen sociodemographic characteristics and exposure types. This is an analytical cross-sectional study and quantitative, based on secondary data from the Viral Hepatitis forms notified by the municipalities of the 17th Health Regional of Paraná from 2015 to 2018. The data were analyzed in the Software Statistical Package for the Social Science for Windows. In the bivariate and multivariable analysis, the prevalence ratios and their respective 95\% confidence intervals were calculated by using Poisson regression with robust adjustment of variance, and a 0,05-significance level. In the multivariable analysis, a statistically significant association was found between the male gender variable and the following exposure types: injectable drugs, inhalant drugs or crack, injectable medicine and three or more sexual partners. Conversely, there was an association between the age group from 18 to 59 years and the following exposure types: inhalant drugs or crack, tattoo and piercing, dental treatment and three or more sexual partners. In the group with low level of education, it was noticed an association between schooling and exposure types, with less prevalence, of the following factors: transfusion and dental treatment. Finally, using inhalant drugs or crack and having three or more partners were exposure factors to $\mathrm{HCV}$ associated with male gender and the age group of 18 to 59 years. Using drugs and injectable medicine were exposure factors associated with males, while tattoo and piercing and dental treatment were associated with the age group up to 59 years.
\end{abstract}

Keywords: Hepatitis C; Hepatitis viral human; Epidemiology; Disease notifications; Public health.

\title{
Resumen
}

El objetivo fue analizar la asociación entre características sociodemográficas y tipos de exposición. Estudio analítico de corte transversal y cuantitativo, con base en datos secundarios de los registros de Hepatitis Virales notificados por los municipios de la $17^{a}$ Regional de Salud del Paraná de 2015 a 2018. En el análisis bivariado como multivariado, las razones de prevalencia y sus intervalos de confianza de $95 \%$ se calcularon mediante regresión de Poisson con ajuste robusto de varianza y un nivel de significancia de 0,05 . En el análisis multivariado se encontró asociación estadísticamente significativa entre la variable sexo masculino y los siguientes tipos de exposición: drogas inyectables, drogas inhalantes o crack, drogas inyectables y tres o más parejas sexuales. Hubo asociación entre el grupo de edad entre 18 y 59 años y los siguientes tipos de exposición: drogas inhalantes o crack, tatuaje y piercing, tratamiento dental y tres o más parejas. En el grupo con bajo nivel educativo, una asociación entre la escolaridad y los tipos de exposición, con menor prevalencia, de los siguientes factores: transfusión y tratamiento odontológico. El uso de drogas inhalantes o crack y tener tres o más parejas fueron factores de exposición al VHC asociados con el sexo masculino y el grupo de edad de 18 a 59 años. El uso de drogas y el uso de medicamentos inyectables fueron factores de exposición asociados con los hombres, mientras que el tatuaje y el piercing y el tratamiento dental se asociaron con el grupo de edad hasta los 59 años.

Palabras clave: Hepatitis C; Hepatitis virales humanas; Epidemiología; Notificación de enfermedades; Salud pública.

\section{Introdução}

Houve diversos avanços quanto ao diagnóstico e tratamento da infecção do vírus da Hepatite C (HCV). Notáveis conquistas que refletiram na prevenção e ao controle, sendo que os mais significativos progressos foram a identificação do agente viral e o desenvolvimento de testes laboratoriais específicos. No entanto, o HCV é considerado um problema de saúde pública mundial pela cronificação, possui prevalência no sexo masculino e na faixa etária de 40 a 59 anos (Ministério da Saúde, 2018a; 2020).

Em virtude, da necessidade de mapear os casos cujo perfil epidemiológico é heterogêneo, como forma de prover subsídios para elaboração de diretrizes de políticas públicas, temos que considerar como situação de risco aqueles que receberam transfusão e ou hemoderivados antes 1993, assim como os que fazem uso de drogas injetáveis, inaláveis ou crack e transmissão por contato direto (seringas com agulhas infectadas, transfusões de sangue e alguns produtos derivados do sangue) e percutâneo (lesões provocadas por instrumentos perfurocortantes) (Ministério da Saúde, 2018b; 2019). 
Globalmente, não se conhece, com precisão, a prevalência do HCV. Estima-se que na América 7,2 milhões de pessoas vivem com o HVC crônica (Organização Pan-Americana da Saúde [OPAS], 2018).

No Brasil, durante o período de 1999 a 2019 foram notificados 384.284 casos de HCV com um dos marcadores sorológicos reagentes. No Paraná (PR) entre 2007 e 2018 foram notificados 13.324 casos, que seguiram o panorama nacional com maiores taxas de detecção no sexo masculino, faixa etária de 40 a 59 anos, seguida da categoria de 20 a 39 anos e 60 anos ou mais, com até 12 anos de estudo (Paraná, 2018; Ministério da Saúde, 2020).

Nessa perspectiva, avaliar o perfil epidemiológico dos casos viabiliza a elaboração de estratégias de saúde voltadas à intervenção para o diagnóstico precoce, controlar surtos o mais breve possível, além de avaliar a eficácia dessas medidas de enfrentamento do HCV (Ministério da Saúde, 2018b). Destaca-se ainda, a necessidade de avaliar os fatores de exposição do HCV nos grupos com maior detecção da doença, como forma de implementar medidas preventivas específicas e direcionadas.

Nesse contexto, objetivo deste estudo foi de analisar a associação entre as características sociodemográficas (sexo, faixa etária e escolaridade) e os tipos de exposição.

\section{Metodologia}

Trata-se de um estudo transversal analítico e quantitativo. No estudo transversal os dados são coletados simultaneamente. As investigações analíticas têm como finalidade avaliar as associações entre exposições e desfechos. Abordagem quantitativa, permite a partir da análise de dados, determinar se existe ou não relação entre variáveis e quantifica o grau dessa associação (Lozada \& Nunes, 2019; Polit \& Beck, 2019; Pereira, Shitsuka, Parreira \& Shitsuka, 2018).

Os dados foram coletados a partir de dados secundários das fichas de Hepatites Virais (HV) do Sistema Nacional de Informação de Agravos de Notificação (SINAN) do Ministério da Saúde. A população de estudo foi constituída por casos notificados/confirmados do HCV, com idade igual ou superior a 18 anos, residentes nos municípios da $17^{a}$ Regional de Saúde do Paraná (RS/PR), em áreas urbanas e rurais com um recorte temporal entre 01 de janeiro de 2015 a 31 de dezembro de 2018. Foram excluídas da amostra os casos de coinfecções por outras HV.

As seguintes variáveis foram categorizadas: idade (18 a 59; 60 ou mais), escolaridade (até 9 anos - ensino fundamental; 10 anos ou mais - ensino médio e superior), raça (branca/não branca), unidade notificadora (hospitalar, Unidade Básica de Saúde - UBS, Centro de Testagem e aconselhamento - CTA/ Centro de Referência Dr. Bruno Piancastelli Filho e outros [Diretoria de Vigilância à Saúde, clínicas, pronto atendimento, hemocentro e não localizado]).

Os municípios de notificação foram categorizados em: pequeno porte (Alvorada do Sul, Assaí, Cafeara, Bela Vista do Paraíso, Centenário do Sul, Florestópolis, Guaraci, Ibiporã, Jaguapitã, Jataizinho, Lupionópolis, Miraselva, Pitangueiras, Porecatu, Prado Ferreira, Primeiro de Maio, Sertanópolis e Tamarana), médio porte (Cambé e Rolândia) e grande porte (Londrina) (Demográfico, 2010).

Para a análise descritiva foram utilizadas frequências absoluta e relativa. Tanto na análise bivariada quanto na multivariável as Razões de Prevalência (RP) e seus respectivos Intervalos de Confiança de 95\% (IC 95\%) foram calculados por meio da regressão de Poisson com ajuste robusto de variância.

No que tange a análise bivariada, considerou-se o cruzamento das variáveis desfecho: drogas injetáveis, drogas inaláveis ou crack, transfusional, hemodiálise, tratamento cirúrgico e dentário, transplante, tatuagem ou piercing, medicamentos injetáveis, acupuntura, três ou mais parceiros sexuais e acidente com material biológico.

As variáveis de exposição foram: sexo, faixa etária e escolaridade. Baseado em critério epidemiológico, as seguintes variáveis foram selecionadas para o modelo multivariável: sexo, faixa etária e escolaridade. Todas as análises foram realizadas 
no programa IBM Software Statistical Package for the Social Science (SPSS) para o Windows e versão 20.0 ${ }^{\circledR}$ (Spss, 2011) adotando um nível de significância de 0,05 .

\section{Resultados}

No período analisado, foram notificados/confirmados 528 casos do HCV, distribuídos no quadriênio da seguinte forma: 136 (25,8\%) casos em 2015, com discreto aumento no ano de 2016, 153 (29,0\%) notificações, em 2017 observou-se uma redução, com 111 (21,0\%) casos e fechando o ano de 2018 com 128 (24,2\%) notificações.

As características demográficas e clínicas dos casos confirmados do HCV estão apresentadas na Tabela 1.

Tabela 1 - Características demográficas e tipos de exposição dos casos confirmados de Hepatite C nos municípios da $17^{a}$ Regional de Saúde, Paraná, Brasil (2015 a 2018).

\begin{tabular}{|c|c|c|}
\hline Variável & $\mathbf{N}$ & $\%$ \\
\hline \multicolumn{3}{|l|}{$\operatorname{Sexo}(n=528)$} \\
\hline Masculino & 293 & 55,5 \\
\hline Feminino & 235 & 44,5 \\
\hline \multicolumn{3}{|l|}{ Idade $(n=528)$} \\
\hline 18 a 59 anos & 339 & 64,2 \\
\hline 60 ou mais & 189 & 35,8 \\
\hline \multicolumn{3}{|l|}{ Escolaridade $^{a}(n=421)$} \\
\hline Até 9 anos & 230 & 54,6 \\
\hline 10 anos ou mais & 191 & 45,4 \\
\hline \multicolumn{3}{|l|}{ Raça/cor ${ }^{a}(n=521)$} \\
\hline Branca & 357 & 68,6 \\
\hline Não branca & 164 & 31,4 \\
\hline \multicolumn{3}{|l|}{ Zona $(n=528)$} \\
\hline Urbana & 515 & 97,6 \\
\hline Rural & 13 & 2,4 \\
\hline \multicolumn{3}{|l|}{ Município de notificação $(n=528)$} \\
\hline Grande porte & 439 & 83,1 \\
\hline Médio porte & 57 & 10,8 \\
\hline Pequeno porte & 32 & 6,1 \\
\hline \multicolumn{3}{|l|}{ Unidade notificadora $(n=528)$} \\
\hline $\mathrm{UBS}^{\mathrm{b}}$ & 244 & 46,2 \\
\hline Hospitalar & 171 & 32,4 \\
\hline $\mathrm{CTA}^{\mathrm{c}}$ & 47 & 8,9 \\
\hline Outros $^{\mathrm{d}}$ & 66 & 12,5 \\
\hline \multicolumn{3}{|l|}{ Relato dos tipos de exposição ${ }^{\mathrm{e}}$} \\
\hline Tratamento dentário $(n=472)$ & 252 & 53,4 \\
\hline Medicamentos injetáveis $(n=511)$ & 233 & 44,1 \\
\hline Tratamento cirúrgico $(n=482)$ & 222 & 42,0 \\
\hline Três ou mais parceiros $(n=467)$ & 127 & 24,1 \\
\hline Tatuagem/piercing $(n=469)$ & 105 & 19,9 \\
\hline Drogas inaláveis ou crack $(n=475)$ & 88 & 16,7 \\
\hline Drogas injetáveis $(\mathrm{n}=478)$ & 80 & 15,2 \\
\hline Transfusional $(\mathrm{n}=491)$ & 78 & 14,8 \\
\hline Acupuntura $(n=471)$ & 32 & 6,1 \\
\hline Hemodiálise $(n=503)$ & 19 & 3,6 \\
\hline Material biológico $(n=501)$ & 12 & 2,3 \\
\hline Transplante $(\mathrm{n}=506)$ & 9 & 1,7 \\
\hline
\end{tabular}

a107 casos não informados/ignorados para escolaridade e 7 casos não informados/ignorados para raça/cor bUnidade Básica de Saúde ${ }^{\mathrm{c} C e n t r o ~ d e ~ T e s t a g e m ~ e ~ a c o n s e l h a m e n t o ~}{ }^{\mathrm{d}}$ Diretoria de Vigilância à Saúde, clínicas, pronto atendimento, hemocentro e não informado. ${ }^{\mathrm{e}}$ As porcentagens não somam $100 \%$ porque cada indivíduo poderia citar várias opções.

Fonte: Autores. 
Observa-se que, a maioria era do sexo masculino (55,5\%), com faixa etária de 18 a 59 anos (64,2\%), baixa escolaridade, de até 9 anos de estudos (54,6\%), da raça branca (68,6\%) e residente na área urbana (97,6\%). Quanto ao município de notificação, prevaleceu o de grande porte $(83,1 \%)$, sendo que a Unidade Básica de Saúde foi a que mais realizou notificações (46,2\%). Entre os tipos de exposição, destacam-se: tratamento dentário $(53,4 \%)$, medicamentos injetáveis $(44,1 \%)$ e tratamento cirúrgico $(42,0 \%)$.

Estão apresentados na Tabela 2 os dados relativos à associação da variável sexo e os tipos de exposição.

Tabela 2 - Associações entre sexo e tipos de exposição dos casos confirmados de Hepatite C pelos municípios da $17^{a}$ Regional de Saúde, Paraná, Brasil (2015 - 2018).

\begin{tabular}{|c|c|c|c|c|c|c|}
\hline \multirow{4}{*}{ SEXO } & \multicolumn{6}{|c|}{ TIPOS DE EXPOSIÇÃO } \\
\hline & \multirow{2}{*}{$\begin{array}{c}\text { TOTAL } \\
\text { (n) }\end{array}$} & \multirow{2}{*}{$\begin{array}{c}\% \\
\text { EXPOSTA }^{a}\end{array}$} & \multicolumn{2}{|c|}{ ANÁLISE BRUTA } & \multicolumn{2}{|c|}{ ANÁLISES MULTIVARIÁVEIS } \\
\hline & & & $\mathbf{R} \mathbf{P}^{\mathbf{b}(\mathrm{IC} 95 \%)}$ & p-VALOR ${ }^{c}$ & $\mathbf{R P}^{\mathbf{d}(\mathrm{IC} 95 \%)}$ & p-VALOR \\
\hline & & & \multicolumn{4}{|c|}{ DROGAS INJETÁVEIS } \\
\hline $\begin{array}{l}\text { Masculino } \\
\text { Feminino }\end{array}$ & $\begin{array}{l}261 \\
217\end{array}$ & $\begin{array}{r}23,8 \\
8,3\end{array}$ & $\begin{array}{c}2,86(1,74-4,68) \\
1\end{array}$ & 0,01 & $\begin{array}{c}2,38(1,41-4,01) \\
1\end{array}$ & $<0,001$ \\
\hline & & & \multicolumn{4}{|c|}{ DROGAS INALÁVEIS OU CRACK } \\
\hline $\begin{array}{l}\text { Masculino } \\
\text { Feminino }\end{array}$ & $\begin{array}{l}258 \\
217\end{array}$ & $\begin{array}{l}24,8 \\
11,1\end{array}$ & $\begin{array}{c}2,24(1,45-3,45) \\
1\end{array}$ & 0,01 & $\begin{array}{c}1,80(1,18-2,76) \\
1\end{array}$ & $<0,001$ \\
\hline & & & \multicolumn{4}{|c|}{ TRANSFUSIONAL } \\
\hline $\begin{array}{l}\text { Masculino } \\
\text { Feminino }\end{array}$ & $\begin{array}{l}275 \\
216\end{array}$ & $\begin{array}{l}13,1 \\
19,4\end{array}$ & $\begin{array}{c}0,67(0,44-1,01) \\
1\end{array}$ & 0,05 & $\begin{array}{c}0,77(0,50-1,18) \\
1\end{array}$ & 0,23 \\
\hline & & & \multicolumn{4}{|c|}{ HEMODIÁLISE } \\
\hline $\begin{array}{l}\text { Masculino } \\
\text { Feminino }\end{array}$ & $\begin{array}{l}281 \\
222\end{array}$ & $\begin{array}{l}5,0 \\
2,3\end{array}$ & $\begin{array}{c}2,21(0,80-6,04) \\
1\end{array}$ & 0,12 & $\begin{array}{c}1,96(0,66-5,86) \\
1\end{array}$ & 0,22 \\
\hline & & & \multicolumn{4}{|c|}{ Tratamento Cirúrgico } \\
\hline $\begin{array}{l}\text { Masculino } \\
\text { Feminino }\end{array}$ & $\begin{array}{l}261 \\
221\end{array}$ & $\begin{array}{l}43,7 \\
48,9\end{array}$ & $\begin{array}{c}0,89(0,73-1,08) \\
1\end{array}$ & 0,25 & $\begin{array}{c}0,98(0,80-1,21) \\
1\end{array}$ & 0,91 \\
\hline & & & \multicolumn{4}{|c|}{ TRATAMENTO DENTÁRIO } \\
\hline Masculino & 257 & 53,3 & $0,99(0,84-1,18)$ & 0,96 & $1,02(0,85-1,22)$ & 0,82 \\
\hline Feminino & 215 & 53,5 & 1 & \multirow{2}{*}{\multicolumn{3}{|c|}{$\begin{array}{c}1 \\
\text { MEDICAMENTOS INJETÁVEIS }\end{array}$}} \\
\hline & & & & & & \\
\hline Masculino & 285 & 50,9 & $1,30(1,07-1,59)$ & 0,08 & $1,29(1,02-1,64)$ & \multirow[t]{2}{*}{0,03} \\
\hline \multirow[t]{2}{*}{ Feminino } & 226 & 38,9 & 1 & & 1 & \\
\hline & & & \multicolumn{4}{|c|}{ TATUAGEM PIERCING } \\
\hline Masculino & 255 & 27,8 & $1,75(1,21-2,52)$ & \multirow[t]{2}{*}{0,03} & $1,39(0,95-2,04)$ & \multirow[t]{2}{*}{0,08} \\
\hline \multirow[t]{2}{*}{ Feminino } & 214 & 15,9 & 1 & & 1 & \\
\hline & & & & & NTURA & \\
\hline Masculino & 256 & 6,2 & $0,84(0,43-01,63)$ & 0,60 & $0,99(0,50-1,94)$ & 0,98 \\
\hline Feminino & 215 & 7,4 & 1 & & 1 & \\
\hline & & & & TRÊS OU M & REIROS SEXUAIS & \\
\hline Masculino & 256 & 37,1 & $2,44(1,71-3,49)$ & 0,01 & $2,18(1,50-3,17)$ & $<0,001$ \\
\hline Feminino & 211 & 15,2 & 1 & & 1 & \\
\hline & & & & Mate & IOLÓGICO & \\
\hline Masculino & 279 & 1,4 & $0,39(0,12-1,30)$ & 0,12 & $0,33(0,08-1,28)$ & 0,11 \\
\hline Feminino & 222 & 3,6 & 1 & & 1 & \\
\hline & & & & & LANTE & \\
\hline Masculino & 281 & 2,1 & $1,60(0,40-6,33)$ & 0,50 & $2,08(0,43-9,91)$ & 0,35 \\
\hline Feminino & 225 & 1,3 & & & 1 & \\
\hline
\end{tabular}

${ }^{\mathrm{a} A s}$ porcentagens não somam $100 \%$ porque cada voluntário poderia marcar várias opções; ${ }^{\mathrm{b}}$ Razão de prevalência; ${ }^{\mathrm{c}} \mathrm{p}$-valor referente a regressão de Poisson com variância robusta; ${ }^{\mathrm{d} A j u s t a d a}$ por: sexo, faixa etária e escolaridade.

Fonte: Autores. 
Verifica-se que, na análise bruta obtiveram associação estatisticamente significativa, com maior frequência no sexo masculino, as variáveis: drogas injetáveis drogas inaláveis, medicamentos injetáveis, tatuagem/piercing e três ou mais parceiros sexuais. Na análise multivariável houve associação entre o sexo e os seguintes fatores de exposição: drogas injetáveis, drogas inaláveis ou crack, medicamentos injetáveis e três ou mais parceiros sexuais, sendo mais frequente no sexo masculino em comparação ao feminino. Em seguida, na Tabela 3, consta os dados relativos à associação da variável idade e os tipos de exposição.

Tabela 3 - Associações entre faixa etária e tipos de exposição dos casos confirmados de Hepatite C pelos municípios da $17^{\mathrm{a}}$ Regional de Saúde, Paraná, Brasil (2015 - 2018).

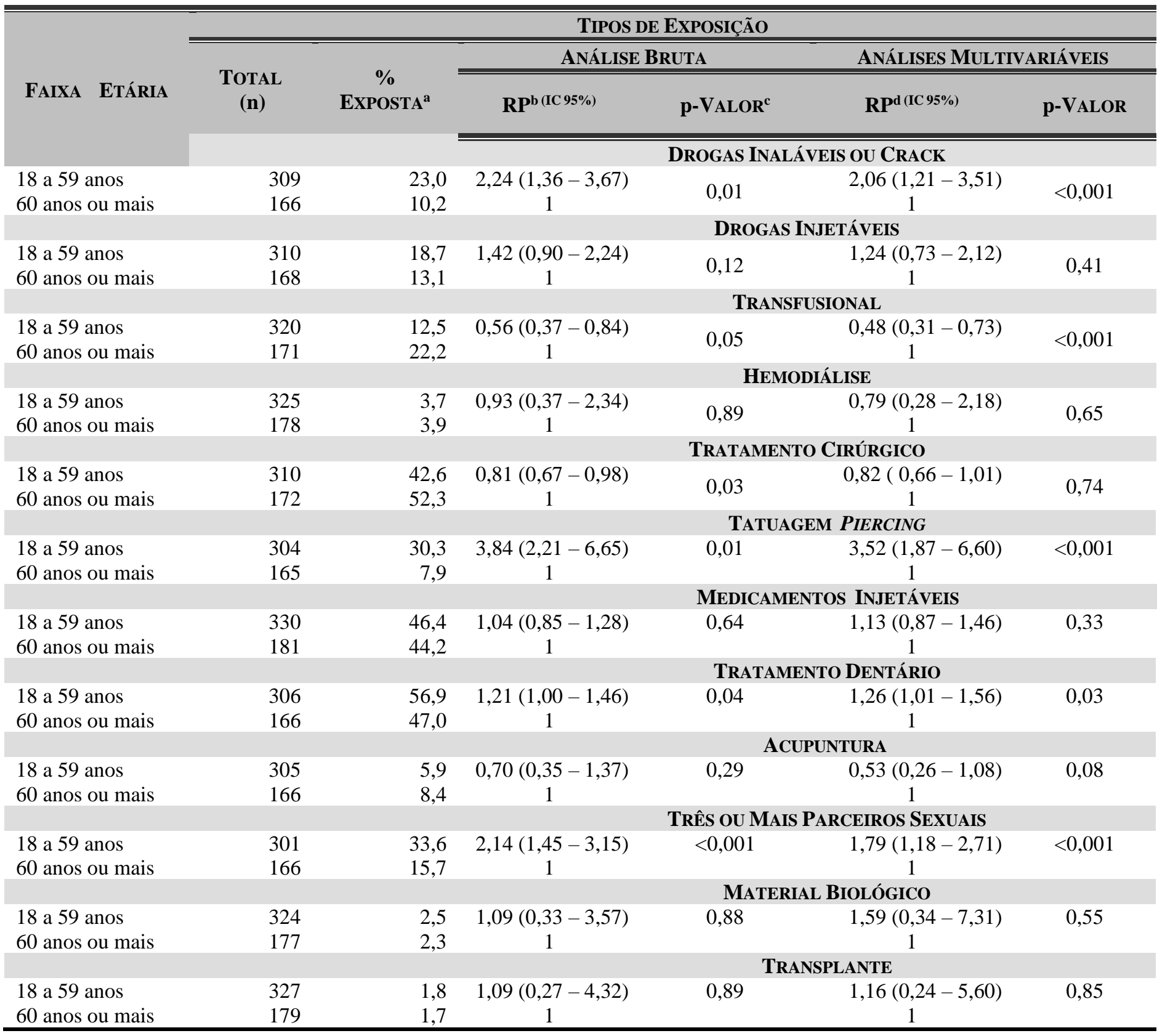

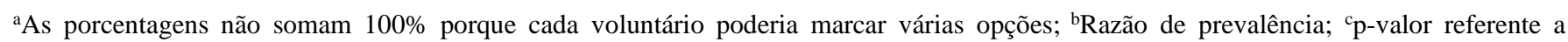
regressão de Poisson; ${ }^{\mathrm{d}}$ Ajustada por: sexo, faixa etária e escolaridade Fonte: Autores. 
Identificou-se que na análise bruta, houve associação estatisticamente significativa com maior ocorrência dos seguintes fatores, na faixa etária de 18 a 59 anos: drogas inaláveis ou crack, tatuagem/piercing, tratamento dentário e três ou mais parceiros. Enquanto na análise multivariável apresentou associação com a idade: drogas inaláveis ou crack, tatuagem e piercing, tratamento dentário e três ou mais parceiros, com maior frequência na faixa etária de 18 a 59 anos.

Na Tabela 4 consta os dados relativos à associação da variável escolaridade e os tipos de exposição.

Tabela 4 - Fatores associados à infecção pelo vírus da Hepatite C entre a escolaridade e os tipos de exposição nos casos confirmados pelos municípios da $17^{\text {a }}$ Regional de Saúde, Paraná, Brasil (2015 - 2018).

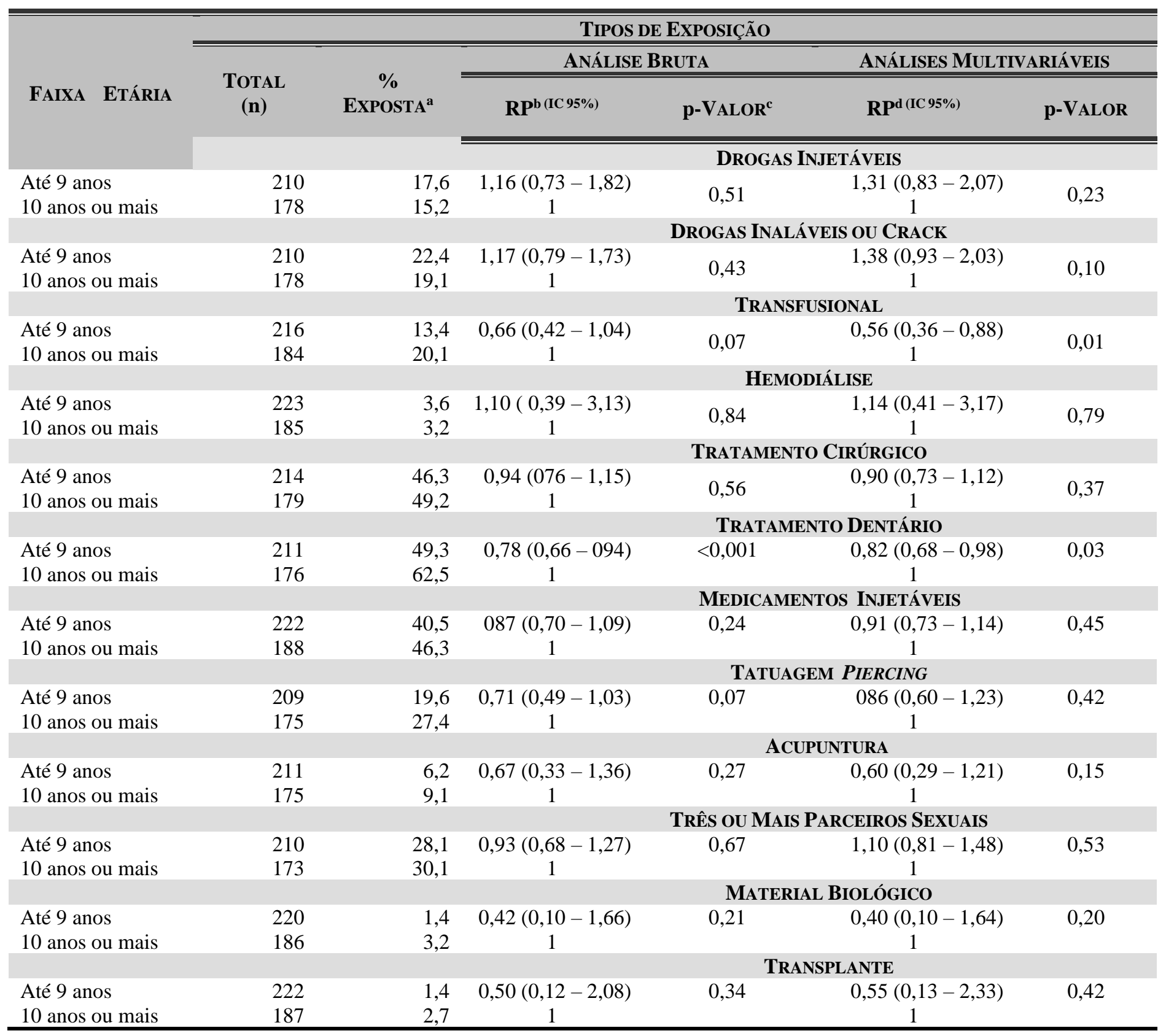

${ }^{\mathrm{a} A s}$ porcentagens não somam $100 \%$ porque cada voluntário poderia marcar várias opções; ${ }^{b}$ Razão de prevalência; ${ }^{\mathrm{c}}$-valor referente a regressão de Poisson; ${ }^{\mathrm{d} A j u s t a d a}$ por: sexo, faixa etária e escolaridade

Fonte: Autores. 
$\mathrm{Na}$ análise bruta houve associação entre escolaridade e tratamento dentário, com menor frequência no grupo até 9 anos de estudo. Enquanto, na análise multivariável, houve menor prevalência, no grupo com baixa escolaridade, dos seguintes fatores de exposição: transfusional e tratamento dentário.

\section{Discussão}

Propôs-se com este estudo analisar a associação entre as características demográficas (sexo, faixa etária e escolaridade) e os tipos de exposição dos casos confirmados com o HCV dos municípios pertencentes a $17^{\mathrm{a}}$ RS/PR.

No presente estudo, houve prevalência do sexo masculino, com faixa etária de 18 a 59 anos e com baixa escolaridade. Estudos sinalizam que indivíduos com essas características, tendem a não se preocuparem com a prevenção de doenças e prática de hábitos saudáveis (Carneiro, Adjuto \& Alves, 2019; Oliveira, Reis, Barreto, Gomes \& Manrique, 2018; Araújo et al., 2020; Oliveira et al., 2020).

Apesar de ser amplamente debatido, ainda não há na literatura estudos aprofundados sobre a suscetibilidade do vírus com o homem. Algumas atitudes podem deixá-los mais vulneráveis a infecção do HCV, como por exemplo, exposição as drogas, múltiplos parceiros sexuais e a baixa procura dos serviços de saúde (Bandeira, Souza, Reis Marques, Peruzini, Guedes \& Souza Neto, 2018; Oliveira, Reis, Barreto, Gomes \& Manrique, 2018; Paraboni, Sbeghen, Wolff \& Moreira, 2012; Demográfico, 2020).

No tocante, faixa etária de 18 a 59 anos, se trata de uma população economicamente ativa, ou seja, que está em período de alta produtividade, por isso podem apresentar mais dificuldades, por exemplo, em faltar um dia de trabalho, para realização de consultas e exames preventivos (Onmt, 2019; Carneiro, Adjuto \& Alves, 2019; Oliveira, Daher, Silva \& Andrade, 2015).

No que tange a análise multivariável, encontrou-se associação estatisticamente significativa com maior prevalência no sexo masculino com as variáveis: drogas injetáveis (RP 2,38; IC 95\% 1,41-4,01) drogas inaláveis ou crack (RP 1,80; IC 95\% 1,18 -2,76), medicamentos injetáveis (RP 1,29; IC 95\% 1,02 - 1,64) e três ou mais parceiros sexuais (RP 2,18; IC 95\%1,50 $3,17)$.

Com relação à faixa etária, naqueles com 18 a 59 anos houve maior razão de prevalência com os seguintes fatores de exposição: drogas inaláveis ou crack (RP 2,06; IC 95\% 1,21 - 3,51), tatuagem e piercing (RP 3,52; IC 95\% 1,87 - 6,60), tratamento dentário (RP 1,26; IC 95\% 1,01 - 1,56), três ou mais parceiros (RP 1,79; IC 95\% 1,18 - 2,71) e menor razão de prevalência entre os casos de 60 anos ou mais na exposição transfusional (RP 0,48; IC 95\% 0,31 - 0,73).

Ressalta-se quanto a escolaridade, houve menor prevalência, no grupo com baixa escolaridade (até 9 anos de estudo) dos fatores de exposição transfusional ( $\mathrm{RP}=0,56$; IC $95 \% 0,36$ - 0,88) e tratamento dentário ( $\mathrm{RP}=0,82$; IC $95 \% 0,68-0,98$ ).

As prevalências de $\mathrm{HCV}$ entre usuários de drogas injetáveis e inaláveis ou crack foram 2,38 e 1,80 vezes, respectivamente superiores no sexo masculino quando comparado ao feminino. $\mathrm{O}$ uso de drogas inaláveis ou crack 2,06 vezes superior entre os indivíduos da faixa etária entre 18 a 59 anos, comparado com aqueles com 60 anos ou mais. Corroborando com este estudo, a United Nations Office on Drugs and Crime -UNODC (2017) estimou que mundialmente quase 12 milhões de pessoas fizeram uso de drogas injetáveis e com isso, mais da metade adquiriram o HCV.

No Brasil, em 2017, foram encontrados dados semelhantes, uma pesquisa realizada pela Fundação Oswaldo Cruz (FIOCRUZ) identificou que 1,4 milhões de pessoas, a maioria do sexo masculino e idade entre 12 e 65 anos já relataram ter feito uso de substâncias ilícitas seja inalável ou injetável, demonstrando assim, comportamento de risco entre os homens e nessa faixa etária para aquisição do HCV (Owens et al., 2020). 
Esses dados sinalizam a necessidade de novas formas de abordagem com esse público, com ações mais efetivas que enfoquem na prevenção, redução de risco, promoção, tratamento, recuperação, reabilitação da saúde e reinserção social (Ministério da Saúde, 2018c; Poder executivo, 2019; Bastos, Vasconcellos, Boni, Reis \& Coutinho, 2017; Gavioli, Pazin, Marangoni, Hungaro, Santana, \& Oliveira, 2020; Owens et al., 2020).

Nesse contexto, destacam-se as ações realizadas em Portugal, que deixou de tratar o uso de drogas como um crime, mas sim como uma questão de saúde. Quando os usuários são identificados com pequenas quantidades de drogas para consumo próprio, é aplicada uma infração administrativa e os usuários são convocados para uma audiência com assistentes sociais, que buscam prevenir que um usuário casual se torne um dependente químico (Kristof, 2017).

No Brasil, a fim de proporcionar a redução de danos nessa população, o MS recomenda a oferta gratuita de seringas, agulhas e a realização de abordagens preventivas sobre a importância do não compartilhamento de objetos perfurantes e acessórios utilizados para o consumo da droga (Ministério da Saúde, 2015; 2017).

No que concerne ao tipo de exposição três ou mais parceiros sexuais, apresentou prevalência superior em 2,18 vezes no sexo masculino e 1,79 vezes na faixa etária de 18 a 59 anos, quando comparados ao sexo feminino e 60 anos ou mais, respectivamente. Embora esta informação tenha sido autodeclarada pelos participantes no momento da notificação, a transmissão por via sexual do HCV é pouco frequente, porém vale lembrar que a ficha de notificação contempla todos os tipos de HV, havendo risco maior para transmissão do vírus da hepatite B (Cassuto et al, 2002).

Cabe destacar que, alguns comportamentos sexuais podem aumentar o risco de infecção pelo HCV, como múltiplos parceiros e relação sexual desprotegida. Esses comportamentos são mais comuns entre os homens e população de jovens entre 15 e 24 anos (Davis et al., 2015; Ministério da Saúde, 2018b; Scull, Keefe, Kafka, Malik \& Kupersmidt, 2020).

É possível que, as políticas de prevenção não estejam impactando essa população de forma efetiva. Ressalta-se, os jovens são fortemente ligados a tecnologia, por isso, o uso da internet e aplicativos móveis podem se tornar ferramentas poderosas nas estratégias de prevenção. Alguns países como Espanha, Estados Unidos, China e Brasil desenvolveram pesquisas sobre essa temática e mostram resultados promissores como o aumento do uso do preservativo e do conhecimento sobre as Infecções Sexualmente Transmissíveis (IST) (Muessig et al, 2015; Besoain, Perez-Navarro, Caylà, Aviñó \& Olalla, 2015; Sun, Stowers, Miller, Bachmann \& Rhodes, 2015; Barbosa, da Silva, de Jesus Mota \& Nichiata, 2019).

Outro comportamento de risco identificado entre os indivíduos investigados, foram tatuagem/piercing, mostrou-se prevalência superior na faixa etária de 18 a 59 anos (3,52 vezes) quando comparado àqueles de 60 anos ou mais. Sabe-se que o risco de transmissão do $\mathrm{HCV}$, é amplamente aumentado quando a tatugem/piercing não são realizados em um ambiente profissional ou quando os materiais são reutilizados de maneira indevida com falhas na limpeza, na desinfecção e esterilização (Patel \& Cobbs, 2016; Agência de Vigilância Sanitária [ANVISA], 2018).

No tocante à exposição por tratamento dentário, observou a prevalência superior ( 1,26 vezes) na faixa etária entre 18 a 59 anos quando comparado com 60 anos ou mais. Em contrapartida, essa exposição foi menor nos indivíduos com até 9 anos de estudo ( 0,82 vezes), ou seja, configurando uma maior prevalência entre os que possuem escolaridade de 10 anos ou mais.

Ressalta-se que, na década de 80, o acesso ao tratamento dentário era condicionado a uma maior renda e escolaridade. Vale ressaltar que a baixa escolaridade vem sendo relacionada a indivíduos que demonstram pouco conhecimento sobre as formas de prevenção e de acesso aos serviços de saúde, incluindo o tratamento dentário (Freitas, Santos, Vasconcelos, Oliveira \& Santos, 2020; Tavares et al., 2016).

Somente, no ano de 2.000 que foi realizado a inclusão da odontologia na Estratégia Saúde da Família (ESF), ampliando o acesso e contribuindo com o princípio de integralidade (Ministério da Saúde, 2000; Guarnizo-Herreño, Tsakos, 
Sheiham, Marmot, Kawachi \& Watt, 2015; Viacava \& Bellido, 2016; Ministério da Saúde, 2018d; Sousa, Henriques, Silva, Severo \& Silva, 2019).

Por fim, não foram encontrados estudos que abordassem sobre associação entre o sexo masculino e medicamentos injetáveis. Sabe-se que a transmissão pode estar atrelada a contaminação dos frascos de medicações com multidoses, com sangue de um paciente infectado que é então transmitido a outro paciente, essa prática já foi muito comum em clínicas de hemodiálise. Em 2001, sete pacientes de um ambulatório dos Estados Unidos contraíram o HCV e as investigações revelaram que a provável fonte de infecção foi a contaminação de um frasco multidose de anestésico (Centers for Disease Control, 2003; Garthwaite, Reddy, Douthwaite, Lines, Tyerman \& Eccles, 2019).

Frente a isso, como estratégia, o Brasil por meio ANVISA vem publicando desde 2003, recomendações sobre a Prevenção de Infecção Relacionada a Corrente Sanguínea e à Assistência à Saúde, sobre o manuseio correto dos frascos de medicamentos multidoses. Esses medicamentos não devem entrar na área de atendimento ao paciente e, se isso ocorrer, devem ser descartados (Centers for Disease Control, 2003; Anvisa, 2003; Anvisa, 2010; 2017; Garthwaite, Reddy, Douthwaite, Lines, Tyerman \& Eccles, 2019).

Cumpre informar que, deve-se ter cautela ao se realizar generalizações para outros cenários. Todavia esta pesquisa poderá trazer contribuições relevantes para saúde pública, no que concerne ao planejamento e à gestão baseado do controle do HCV na Atenção Primária à Saúde (APS).

Como limitação deste estudo foram a falta de acesso aos dados de coinfecção por outras IST, comorbidades e informações socioeconômicas nos registros do SINAN nos anos de 2015 a 2018. Reitera-se, a necessidade do MS instituir uma ficha do SINAN específica para a investigação e confirmação dos casos do HCV.

Entretanto, os resultados apontados neste estudo poderão servir como orientação para gestores e profissionais de saúde, em relação aos locais prioritários que necessitam de maior atenção na luta contra o HCV, por muitas vezes evitando óbitos. Desta forma, este trabalho aponta aspectos relevantes a serem considerados sobre esta realidade, tanto sob o ponto de vista clínico, quanto à efetividade de estratégias para reduzir as ocorrências da doença.

\section{Conclusão}

Verificou-se, neste estudo, que o perfil epidemiológico dos 528 casos do HCV dos municípios pertencentes a $17^{\mathrm{a}}$ RS/PR, com predomínio do sexo masculino (55,5\%), faixa etária de 18 a 59 anos $(64,2 \%)$, baixa escolaridade $(54,6 \%)$ e da raça/cor branca $(67,6 \%)$.

No que tange a análise multivariável, encontrou-se associação estatisticamente significativa com maior prevalência no sexo masculino das variáveis: drogas injetáveis, drogas inaláveis ou crack, medicamentos injetáveis e três ou mais parceiros sexuais.

Com relação à faixa etária, a categoria com idade entre 18 a 59 anos houve maior prevalência dos fatores de exposição: drogas inaláveis ou crack, tatuagem e piercing, tratamento dentário e três ou mais parceiros. Considerando-se a escolaridade, houve menor prevalência, no grupo com baixa escolaridade (até 9 anos de estudo) dos fatores de exposição transfusional e tratamento dentário.

Assim sendo, os municípios pertencentes a $17^{\mathrm{a}} \mathrm{RS} / \mathrm{PR}$ investigados, é necessária a adoção de estratégias que visem melhorar o controle do HCV e reduzir sua disseminação e, consequentemente, o número de óbitos ocasionados pela doença e ainda, risco elevado de cronificação. 
Diante do exposto, sugerem-se novos estudos sobre essa temática envolvendo outras localidades, objetivando um maior conhecimento do cenário, com definições de estratégias de intervenção mais eficazes para prevenção e controle desse agravo em nível estadual e nacional.

\section{Referências}

Anvisa. Agência Nacional de Vigilância Sanitária (BR). (2003). Resolução RDC n. ${ }^{\text {45, de }} 12$ de março de 2003. Dispõe sobre o Regulamento Técnico de Boas Práticas de Utilização das Soluções Parenterais em Serviços de Saúde.

Anvisa. Agência Nacional de Vigilância Sanitária (BR). (2010). Orientações para prevenção de infecção primária de corrente sanguínea.

Agência Nacional de Vigilância Sanitária (BR). (2018). Relatório Preliminar de Análise de Impacto Regulatório sobre Processamento de Dispositivos Médicos.

Araújo, A. I. N., de Figueirêdo Oséas, J. M., de Faria, J. C. B., Mendonça, B. D. P. N., Lima, C. M., Leite, F. P. P., \& de Melo, L. A. (2020). Perfil epidemiológico das hepatites bec no estado do rio grande do norte. Revista Ciência Plural,6(3), 35-52. https://periodicos.ufrn.br/rcp/article/download/20537/13264/

Bandeira, L. L. B., de Souza, C. S., dos Reis Marques, D., Peruzini, G. A., Guedes, L. V., \& de Souza Neto, J. D. (2018). Epidemiologia das hepatites virais por classificação etiológica. Revista da Sociedade Brasileira de Clínica http://www.sbcm.org.br/ojs3/index.php/rsbcm/article/view/376

Barbosa, B. J. P., da Silva, A. P., de Jesus Mota, T., \& Nichiata, L. Y. I. (2019). Análise do conteúdo central dos aplicativos sobre HIV para smartphones. Journal of Health Informatics, 11(1). http://www.jhi-sbis.saude.ws/ojs-jhi/index.php/jhi-sbis/article/view/653

Bastos, F. I. P. M., Vasconcellos, M. T. L. D., De Boni, R. B., Reis, N. B. D., \& Coutinho, C. F. D. S. (2017). III levantamento nacional sobre o uso de drogas pela população brasileira. https://www.arca.fiocruz.br/handle/icict/34614

Besoain, F., Perez-Navarro, A., Caylà, J. A., Aviñó, C. J., \& de Olalla, P. G. (2015). Prevention of sexually transmitted infections using mobile devices and ubiquitous computing. International journal of health geographics, 14(1), 18. https://doi.org/10.1186/s 12942-015-0010-z

Carneiro, V. S. M., Adjuto, R. N. P., \& Alves, K. A. P. (2019). Saúde do homem: identificação e análise dos fatores relacionados à procura, ou não, dos serviços de atenção primária. Arquivos de Ciências da Saúde da UNIPAR, 23(1). https://doi.org/10.25110/arqsaude.v23i1.2019.6521

Cassuto, N. G., Sifer, C., Feldmann, G., Bouret, D., Moret, F., Benifla, J. L., \& Poncelet, C. (2002). A modified RT-PCR technique to screen for viral RNA in the semen of hepatitis C virus-positive men. Human Reproduction, 17(12), 3153-3156. https://doi.org/10.1093/humrep/17.12.3153

Centers for Disease Control (US), Centers for Disease Control, \& Prevention (US). (2002). Morbidity and mortality weekly report: MMWR (Vol. 51, No. 3853). US Department of Health, Education, and Welfare, Public Health Service, Center for Disease Control.

Davis, K. C., Danube, C. L., Neilson, E. C., Stappenbeck, C. A., Norris, J., George, W. H., \& Kajumulo, K. F. (2016). Distal and proximal influences on men's intentions to resist condoms: Alcohol, sexual aggression history, impulsivity, and social-cognitive factors. AIDS and Behavior, $20(1), 147-157$.

Freitas, J. L. G. da S., Santos, L. V., Vasconcelos, C. R. P., Oliveira, V. M., \& dos Santos, S. M. P. (2020). Analysis of assistance in sexually transmitted infections primary care. Research, Society and Development, 9(9), e946998009. https://doi.org/10.33448/rsd-v9i9.8009

Garthwaite, E., Reddy, V., Douthwaite, S., Lines, S., Tyerman, K., \& Eccles, J. (2019). Clinical practice guideline management of blood borne viruses within the haemodialysis unit. BMC nephrology, 20(1), 388.

Gavioli, A., Pazin, P. T. N., Marangoni, S. R., Hungaro, A. A., Santana, C. J., \& Oliveira, M. L. F. D. (2020). Consumo de drogas por homens internados em hospital psiquiátrico. Revista Latino-Americana de Enfermagem, 28. http://dx.doi.org/10.1590/1518-8345.3370.3296

Guarnizo-Herreño, C. C., Tsakos, G., Sheiham, A., Marmot, M. G., Kawachi, I., \& Watt, R. G. (2015). Austin Powers bites back: a cross sectional comparison of US and English national oral health surveys. bmj, 351. https://doi.org/10.1136/bmj.h6543

Demográfico, I. C. (2010). Instituto Brasileiro de Geografia e Estatística Cidades. https://cidades.ibge.gov.br/

Demográfico, I. C. (2020). Instituto Brasileiro de Geografia. Ministério da Saúde. Ministério da Economia. Informações sobre domicílios, acesso e utilização dos serviços de saúde: Brasil, Grandes Regiões e Unidades da Federação. Pesquisa Nacional de Saúde. https://biblioteca.ibge.gov.br/visualizacao/livros/liv101748.pdf.

Kristof, N. (2017). The Ne Work Times. How to Win a War on Drugs. https://idhdp.com/media/531831/how-to-win-a-war-on-drugs-the-new-york-times.pdf.

Lozada, G., Nunes, K. S. (2019). Metodologia Científica: Sagah Educação.

Ministério da Saúde. (2000). Portaria n $n^{\circ}$ 1444, de 28 de dezembro de 2000. Brasília. 
Ministério da Saúde. Secretaria de Vigilância em Saúde. Departamento de Vigilância, Prevenção e Controle das Infecções Sexualmente Transmissíveis, do HIV/Aids e das Hepatites Virais. (2015). Manual Técnico para o Diagnóstico das Hepatites Virais. Brasília. http://www.aids.gov.br/sites/default/files/pub/ 2015/57798/manual_tecnico_hv_pdf_75405.pdf

Ministério da Saúde. Secretaria de Vigilância em Saúde. Departamento de Vigilância, Prevenção e Controle das Infecções Sexualmente Transmissíveis, do HIV/Aids e das Hepatites Virais. (2017). Prevenção combinada do HIV bases conceituais para profissionais, trabalhadores(as) e gestores(as) de saúde. Brasília. http://www.aids.gov.br/pt-br/pub/2017/prevencao-combinada-do-hiv-bases-conceituais-para-profissionais-trabalhadoresas-e-gestores.

Ministério da Saúde. Secretaria de Vigilância em Saúde. Departamento de Vigilância, Prevenção e Controle das Infecções Sexualmente Transmissíveis, do HIV/Aids e das Hepatites Virais. (2018a). Manual Técnico para o Diagnóstico das Hepatites Virais. Brasília. http://www.aids.gov.br/pt-br/pub/2015/manualtecnico-para-o-diagnostico-das-hepatites-virais

Ministério da Saúde. Secretaria de Vigilância em Saúde. Departamento de Vigilância, Prevenção e Controle das Infecções Sexualmente Transmissíveis, do HIV/Aids e das Hepatites Virais. (2018b). Plano para Eliminação da Hepatite C no Brasil. Brasília. http://www.aids.gov.br/system/tdf/pub/2016/66284/ plano_para_eliminacao_hepatite_c_no_brasil_170119.pdf?file=1\&type=node\&id=66284\&force $=1$.

Ministério da Saúde. Secretaria de Atenção à Saúde. (2018c). Glossário Temático: Saúde do Homem. Brasília. http://bvsms.saude.gov.br/bvs/ publicacoes/glossario_tematico_saude_homem.pdf.

Ministério da Saúde. Secretaria de Atenção à Saúde. Departamento de Atenção Básica. (2018d). A Saúde Bucal no Sistema Único de Saúde. Brasília. https://portalarquivos2. saude.gov.br/images/pdf/2019/junho/25/guia-vigilancia-saude-volume-unico-3ed.pdf.

Ministério da Saúde. Secretaria de Atenção à Saúde. (2019). Guia de Vigilância em Saúde. Brasília. https://portalarquivos2.saude.gov.br/images/ pdf/2019/junho/25/guia-vigilancia-saude-volume-unico-3ed.pdf

Ministério da Saúde. Secretaria de Atenção à Saúde. Boletim epidemiológico. (2020). Hepatites Virais 2020. Brasília. http://www.aids.gov.br/ptbr/pub/2020/boletim-epidemiologico-hepatites-virais-2020.

Muessig, K. E., Bien, C. H., Wei, C., Lo, E. J., Yang, M., Tucker, J. D., ... \& Hightow-Weidman, L. B. (2015). A mixed-methods study on the acceptability of using eHealth for HIV prevention and sexual health care among men who have sex with men in China. Journal of medical Internet research, 17(4), e100. https://doi.org/10.2196/jmir.3370

Oliveira, M. M. D., Daher, D. V., Silva, J. L. L. D., \& Andrade, S. S. C. D. A. (2015). A saúde do homem em questão: busca por atendimento na atenção básica de saúde. Ciência \& Saúde Coletiva, 20(1), 273-278. https://doi.org/10.1590/1413-81232014201.21732013

Oliveira, T. J. B., Reis, L. A. P. D., Barreto, L. D. S. L. O., Gomes, J. G., \& Manrique, E. J. C. (2018). Perfil epidemiológico dos casos de hepatite C em um hospital de referência em doenças infectocontagiosas no estado de Goiás, Brasil. Revista Pan-Amazônica de Saúde, 9(1), 51-57. ttp://dx.doi.org/10.5123/s2176-62232018000100007

Oliveira, E. H. de, Holanda, E. C., Almeida, A. J. A. de, Verde, R. M. C. L., Sousa, F. das C. A., Andrade, S. M. de, \& Cunha, M. A. (2020). Clinical and epidemiological aspects of hepatitis $\mathrm{C}$ cases in the State Of Maranhão, Brazil. Research, Society and Development,9(7), e120973720. https://doi.org/10.33448/rsd-v9i7.3720

Onmt. Observatório Nacional de Mercado de Trabalho (2019). Boletim das políticas públicas de emprego, trabalho e renda. http://portalfat.mte.gov.br/wpcontent/uploads/2019/07/Boletim-de-Pol\%C3\%ADticas-P\%C3\%BAblicas-de-Emprego-Trabalho-e-Renda-2\%C2\%BA-Trimestre-Atualizado-CTPS.pdf.

Opas. Organização Pan-Americana da Saúde. Organização Mundial da Saúde. (2018). Aumento do acesso a tratamento para hepatites poderá salvar milhões de vidas nas Américas. https://www.paho.org/bra/index.php?option=com _content\&view=article\&id=5723:aumento-do-acesso-a-tratamento-para-hepatitespodera-salvar-milhares-de-vidas-nas-americas\&Itemid=812.

Owens, D. K., Davidson, K. W., Krist, A. H., Barry, M. J., Cabana, M., Caughey, A. B., \& Wong, J. B. (2020). Screening for hepatitis C virus infection in adolescents and adults: US Preventive Services Task Force recommendation statement. Jama, 323(10), 970-975. Doi:10.1001/jama.2020.1123

Paraboni, M. L. R., Sbeghen, M. D., Wolff, F. H., \& Moreira, L. B. (2012). Risk factors for infection with different hepatitis C virus genotypes in southern Brazil. The Scientific World Journal, 2012. https://doi.org/10.1100/2012/946954

Paraná. Secretaria de Estado da Saúde. Divisão de Infecções Sexualmente Transmissíveis, Hepatites Virais e Tuberculose. (2018). Boletim epidemiológico Hepatites Virais. Curitiba. http://www.saude.pr.gov.br/sites/ default/arquivos_restritos/files/documento/2020-04/boletimhepatitesvirais2018.pdf.

Patel, M., \& Cobbs, C. G. (2016). Infections from Body Piercing and Tattoos. Infections of Leisure, 307-323. https://doi.org/10.1128/9781555819231.ch15

Pereira, A. S., Shitsuka, D. M., Parreira, F. J., \& Shitsuka, R. (2018). Metodologia da pesquisa científica. https://repositorio.ufsm.br/bitstream/handle/1/15824/Lic_Computacao_Metodologia-Pesquisa-Cientifica.pdf?sequence=1

Poder executivo. Diário Oficial da União. Atos do Poder Executivo. (2019). Decreto $n^{o}$ 9.761, de 11 de Abril de 2019. Brasília. https://www.in.gov.br/materia// asset_publisher/Kujrw0TZC2Mb/content/id/71137357/do1e-2019-04-11-decreto-n-9-761-de-11-de-abril-de-2019-71137316

Polit, D. F., Beck, C. T. (2019) Fundamentos de Pesquisa em Enfermagem. (Vol. 9): Artmed editora.

Scull, T. M., Keefe, E. M., Kafka, J. M., Malik, C. V., \& Kupersmidt, J. B. (2020). The understudied half of undergraduates: risky sexual behaviors among community college students. Journal of American college health, 68(3), 302-312. https://doi.org/10.1080/07448481.2018.1549554

Sousa, J. L. D., Henriques, A., Silva, Z. P. D., Severo, M., \& Silva, S. (2019). Posição socioeconômica e autoavaliação da saúde bucal no Brasil: resultados da Pesquisa Nacional de Saúde. Cadernos de Saúde Pública, 35, e00099518. https://doi.org/10.1590/0102-311X00099518 
Research, Society and Development, v. 10, n. 1, e34110111869, 2021

(CC BY 4.0) | ISSN 2525-3409 | DOI: http://dx.doi.org/10.33448/rsd-v10i1.11869

Spss, I. I. B. M. (2011). IBM SPSS statistics for Windows, version 20.0. New York: IBM Corp, 440.

Sun, C. J., Stowers, J., Miller, C., Bachmann, L. H., \& Rhodes, S. D. (2015). Acceptability and feasibility of using established geosocial and sexual networking mobile applications to promote HIV and STD testing among men who have sex with men. AIDS and Behavior, 19(3), 543-552. https://link.springer.com/article/10.1007/ s10461-014-0942-5

Tavares, N. U. L., Bertoldi, A. D., Mengue, S. S., Arrais, P. S. D., Luiza, V. L., Oliveira, M. A., ... \& Pizzol, T. D. S. D. (2016). Fatores associados à baixa adesão ao tratamento farmacológico de doenças crônicas no Brasil. Revista de Saúde Pública, 50, 10s. https://doi.org/10.1590/S1518-8787.2016050006150

Unodc. United Nations Office on Drugs and Crime. Persons held in prison (2018). https://dataunodc.un.org/data/Prison/Persons \%20held\%20in \%20prison.

Viacava, F., \& Bellido, J. G. (2016). Condições de saúde, acesso a serviços e fontes de pagamento, segundo inquéritos domiciliares. Ciência \& Saúde Coletiva, 21, 351-370. https://doi.org/10.1590/1413-81232015212.19422015 